\title{
Diffusion Coefficient Modeling of a Silicon Solar Cell under Irradiation Effect in Frequency: Electric Equivalent Circuit
}

\author{
${ }^{1}$ Ibrahima TALL, ${ }^{2}$ Boureima SEIBOU, ${ }^{1}$ Mohamed Abderrahim Ould El Moujtaba , ${ }^{1}$ Amadou DIAO, \\ ${ }^{3}$ Mamadou WADE, ${ }^{1}$ Grégoire SISSOKO \\ (1) Laboratory of Semiconductors and Solar Energy, Physics Department, Faculty of Science and \\ Technology, University Cheikh Anta Diop, Dakar, Senegal \\ . (2) Mines, Industry and Geology School of Niamey- Niger \\ (3) Electromechanical Engineering Department, Polytechnic School of Thies - Sénégal
}

\section{Abstract:}

In this paper, a theory on the determination of the diffusion coefficient of the excess minority carriers of a silicon solar cell is presented. The expression of the diffusion coefficient, related to the modulation frequency, the irradiation energy and the damage coefficient is studied and then performed by using the impedance spectroscopy method and Bode and Nyquist diagrams. Based on the diffusion coefficient, we deduce the diffusion length, the cutoff frequency and some electrical parameters obtained from the equivalent circuits of the diffusion coefficient.

Keywords: Diffusion coefficient, irradiation energy, frequency modulation, solar cell.

\section{INTRODUCTION}

Photovoltaic effect is ensured by a photovoltaic cell which energy conversion depends on the nature of the semiconductor structure its technique of manufacturing and operating points. In this study, we will be interested in one of the intrinsic or phenomenological parameters of a silicon solar cell, namely the diffusion coefficient of the minority carriers in the frame. This parameter which represents the diffusion of the minority carriers is quite important for the solar cell output. Moreover, several studies were made on the diffusion coefficient $[1,2]$ and the diffusion length of the minority carriers $[3,4]$ in the goal of improving the photovoltaic cells quality for a better energy output. Our study is reported to the minority carriers' diffusion coefficient determination by the Fick's low in the base region of a silicon solar cell frequency modulation and irradiation energy. From this, an expression of the diffusion coefficient is established according to the frequency and the irradiation energy. Thereby, we note that, as the frequency modulation effect, the irradiation energy also changes the behavior of the diffusion coefficient where capacitive effects appear. Considering the diffusion of the minority carriers in the base as an electrical phenomena, an analogy of electric-diffusion, by means of both Bode and Nyquist diagrams [5] diffusion coefficient, is laid out. An equivalent circuit of the diffusion coefficient is then deduced and the related parameters were calculated.

\section{THEORY}

On the figure1, an $\mathrm{n}^{+}-\mathrm{p}-\mathrm{n}^{+}$type of a silicon solar cell with a parallel vertical junction $[6,7]$ is presented:

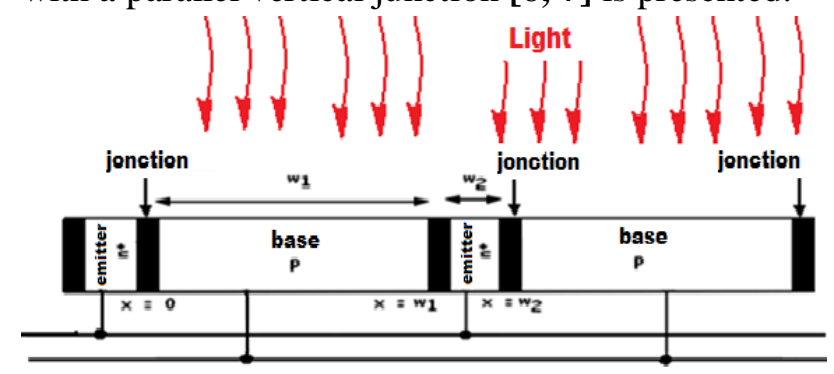

Figure1: Structure of an $\mathbf{n}^{+}-\mathbf{p}-\mathbf{n}^{+}$type of a solar cell with parallel vertical junction.

Illumination of a solar cell under frequency and irradiation, creates electron-hole pairs generation in the base. These electrons and holes as called minority carriers, will diffuse themselves in the frame. The diffusion of an impurity in a solid is a slow physical phenomenon, activated thermically and depends on several factors like the temperature and the thermal processing time, the concentration of the impurity considered, the atmosphere of 
annealing etc...It is represented by a diffusion coefficient D (generally expressed in $\mathrm{cm} . \mathrm{s}^{-1}$ ) itself described by the first and the second Fick's law, respectively associated the flow of particles of concentration $\mathrm{C}(\mathrm{x}, \mathrm{T})$ in the presence of a gradient of concentration (equation 1) and with the conservation of the species (equation 2) [ 8 ].

$$
\begin{aligned}
& J \boldsymbol{\psi}, t_{-}=-D \ll \frac{\partial C \boldsymbol{\psi}, t^{-}}{\partial x} \\
& \frac{\partial C \varangle, t^{-}}{\partial t}=-\frac{\partial J \ll, t^{-}}{\partial x}
\end{aligned}
$$

Combination of equations 1 and 2 conduit with the general equation of the diffusion (equation 3 )

$$
\frac{\partial C \ll, t]}{\partial t}=\frac{\partial}{\partial x}\left[D \ll \frac{\partial C \ll, t]}{\partial x}\right]
$$

It should be noted that during the generalization of the equation (3) a three dimension, the coefficient of diffusion is a tensor of rank two, but in the particular case of the cubic systems and in particular in the nonconstrained Silicon and SiGe alloys, he becomes a scalar size [9].Moreover, the equation (3) is only one "simplified version" of the equation of the diffusion. Indeed, of the physical phenomena of different nature (gradients of electric potential, of temperature, chemical...) and being able to have a dominating action on the diffusivity of the atomic species are not taken here into account [8]. When the coefficient of diffusion is independent of the concentration (case generally observed in the intrinsic semiconductors), the equation of the diffusion is summarized with a partial derivative equation of the second order of the concentration of the migrant species. However, as we will see it during this work, this property will not be checked in our case of study and the resolution of the equation of the diffusion will require a numerical integration. There are several methods of determination of the diffusion coefficient. Generally, the movement of the impurities in a crystal, described by their coefficient of diffusion, can be expressed in the form of Arrhenius' law (equation 4) where $\mathrm{E}_{\mathrm{a}}, \mathrm{D}_{0}, \mathrm{~K}$ and $\mathrm{T}$ are respectively the activation energy the pre-factor of the diffusion coefficient, the Boltzmann constant and the absolute temperature expressed in Kelvin.

$$
D=D_{0} \exp \left(-\frac{E_{a}}{k T}\right)
$$

The diffusion of minority carriers in the base of the solar cell is related to their lifetime which expression is given as below [10],

$$
\frac{1}{\tau}=\frac{1}{\tau_{\mathrm{O}}}+k l \cdot \phi_{p}
$$

In this equation, $\mathrm{Kl}$ is the damage coefficient, $\phi_{\mathrm{p}}$ the irradiation energy, $\tau_{0}$ the average lifetime of the minority carriers without irradiation. The expressions of diffusion length and diffusion coefficient according to the irradiation energy and the damage coefficient are given respectively by equations (6) and (7):

$$
\begin{aligned}
& L \ll l, \phi_{p}=\sqrt{\frac{1}{\frac{1}{L_{o}^{2}}+k l \cdot \phi_{p}}} \\
& D \ll l, \phi_{p}=\frac{L \ll l, \phi_{p}^{2}-}{\tau}
\end{aligned}
$$

Where $L_{o}$ is the minority carriers diffusion length without irradiation energy or damage coefficient. Considering the continuity equation of the minority carriers in the base of the solar cell [11].

$D\left(k l, \phi_{p}\right) \frac{\partial^{2} \delta(x, t)}{\partial x^{2}}-\frac{\delta(x, t)}{\tau}=-G(z, t)+\frac{\partial \delta(x, t)}{\partial t}$

We get the complex diffusion length:

$$
L_{\phi}=L\left(l, \phi_{p} \cdot \sqrt{\frac{1-j \cdot \omega \cdot \tau}{1+\epsilon . \omega^{2}}}\right.
$$

and the complex diffusion coefficient according to angular frequency:

$D_{\phi}=D\left(l, \phi_{p}\left\{\left[\frac{1+\omega^{2} \cdot \tau^{2}}{\left(-\omega^{2} \cdot \tau^{2}\right)+\left(\omega \cdot \tau^{\nu}\right)}+\omega \cdot \tau \cdot \frac{-\omega^{2} \cdot \tau^{2}-1}{\left(-\omega^{2} \cdot \tau^{2}\right)+\left(\omega \cdot \tau^{2}\right)} \cdot j\right]\right.\right.$

(10)

With $L\left(p, k l, \phi_{p} \overline{\bar{\gamma}} L_{\phi}\right.$ and $D\left(, k l, \phi_{p} \overline{\bar{\gamma}} D_{\phi}\right.$ $\omega=2 \pi f$ is the angular frequency and $f$ the modulation frequency. 


\section{DIFFUSION COEFFICIENT PROFILE}

In Figure 2, the diffusion coefficient according to $\log (\omega)$, for different values of the irradiation energy, is represented:

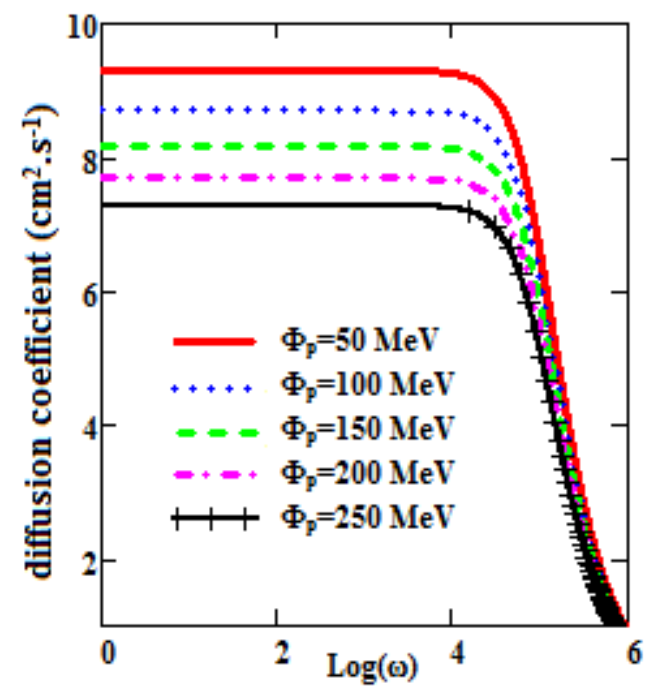

Figure 2: diffusion coefficient versus $\log (\omega)$, $\mathrm{KI}=15 \mathrm{~cm}^{2} \cdot \mathrm{s}^{-1}$.

For a given value of irradiation energy, in the range of the angular frequency $\left[0 \mathrm{rad} . \mathrm{s}^{-1} ; 10^{4} \mathrm{rad}_{\mathrm{s}} \mathrm{s}^{-1}\right]$ as we are in quasi steady state, the diffusion coefficient remains constant. But in the range of the angular frequency $\left[10^{4} \mathrm{rad}_{\mathrm{s}} \mathrm{s}^{-1} ; 10^{6} \mathrm{rad} . \mathrm{s}^{-1}\right]$, the diffusion coefficient decreases drastically with frequency. The higher frequencies do not permit to minority carriers to diffuse because many of them will be recombined either in bulk or in surface of the solar cell. We note that the increase of irradiation energy, decreases the amplitude of diffusion coefficient since the solar cell properties are damaged by affecting also electrical parameters.

\section{PROFILE OF THE DIFFUSION COEFFICIENT ACCORDING TO THE ENERGY OF IRRADIATION}

With the figure (3), we represent the profile of the diffusion coefficient according to the energy of irradiation for various values of the frequency:

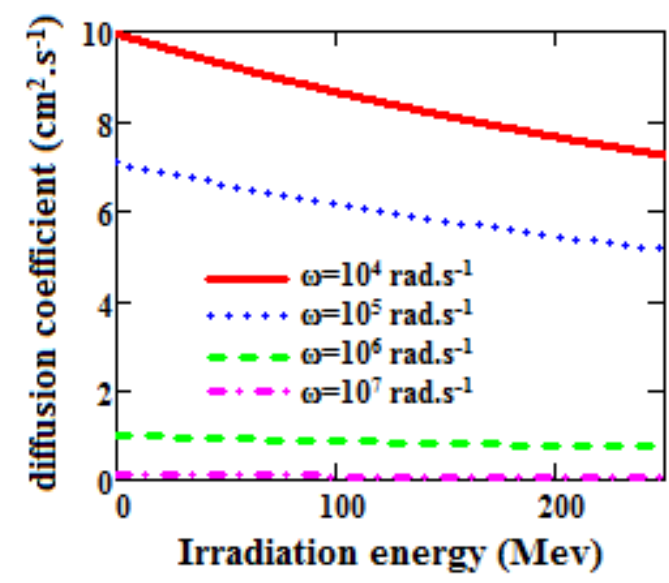

Figure 3: coefficient of diffusion according to the flow of irradiation for various frequencies. $K \mathrm{l}=10 \mathrm{~cm}^{2} / \mathrm{S}$ We can notice that the diffusion coefficient decreases with the frequency modulation; in fact, for a higher frequency (starting from $10^{6}$ rad.s $^{-1}$ ), the carriers cannot be released any more so that they cannot move correctly into the material. Since the diffusion coefficient expresses the capacity of the carriers to be spread into the material.

\section{BODE DIAGRAM OF THE DIFFUSION COEFFICIENT}

The logarithm of the diffusion coefficient module and its phase are represented according to the logarithm of the angular frequency, for different values of the irradiation energy, on figures 3-a) and 3-b) respectively:

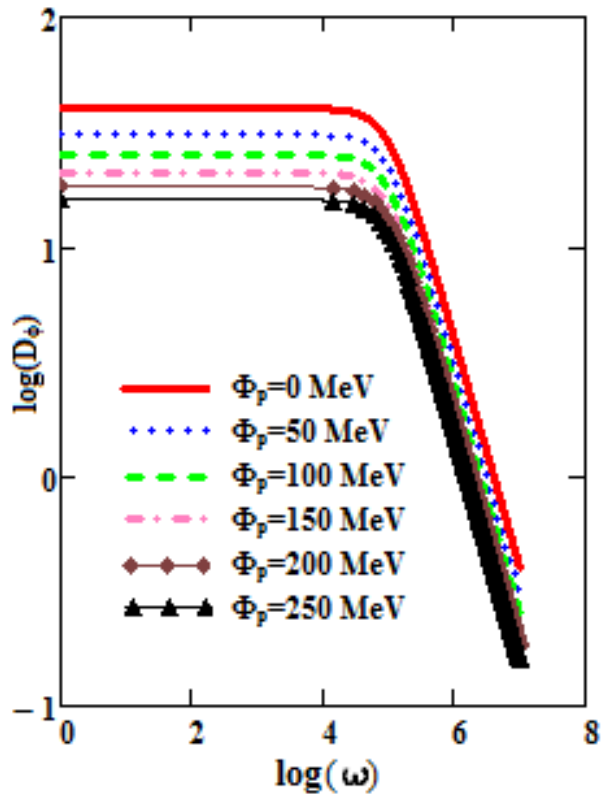

(a) 


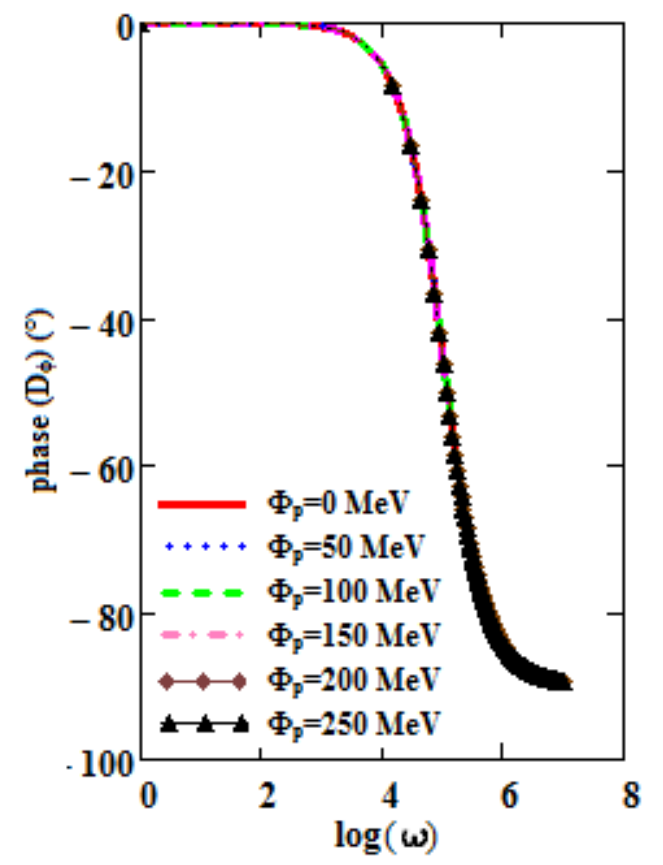

(b)

Figure 4: $\log \left(\mathrm{D}_{\phi}\right)$ and its phase versus $\log (\omega)$, $\mathrm{Kl}=15 \mathrm{~cm}^{2} \cdot \mathrm{s}^{-1}$

In figure 3-a), we obtain the same curves as in figure 2 . In the range of the angular frequency [0 rad.s ${ }^{-1} ; 10^{4}$ rad. $s^{-1}[$, the phase is practically equal to zero: the diffusion of the photo generated minority carriers in the base of the solar cell appears simultaneously with the optical excitation. For higher frequencies we have a negative phase shift that shows the capacitive effect of the diffusion coefficient. There is a delay between optical excitation and the generated minority carriers' diffusion. This diffusion coefficient behavior is similar to the capacitance one; that's why we consider the diffusion phenomenon as a plan capacitor. We note that the phase is not affected by the irradiation energy. One notes, for a given curve of the diffusion coefficient logarithm versus $\log (\omega)$, there is an angular frequency corresponding to a change of the curve, called the cut-off angular frequency $\omega_{\mathrm{c}}\left(\right.$ here $\left.\omega_{\mathrm{c}}=10^{5} \mathrm{rad} . \mathrm{s}^{-1}\right)$ which can lead to the determination of the minority carriers lifetime in the base of the solar cell. To determine $\omega_{c}$ graphically, one projects the intercepted point of the two tangents lines to the curve (see figure 4 ) on the $\log (\omega)$ axis. This projection permits us to know the $\log \left(\omega_{\mathrm{c}}\right)$ value which leads to $\omega_{\mathrm{c}}$ :

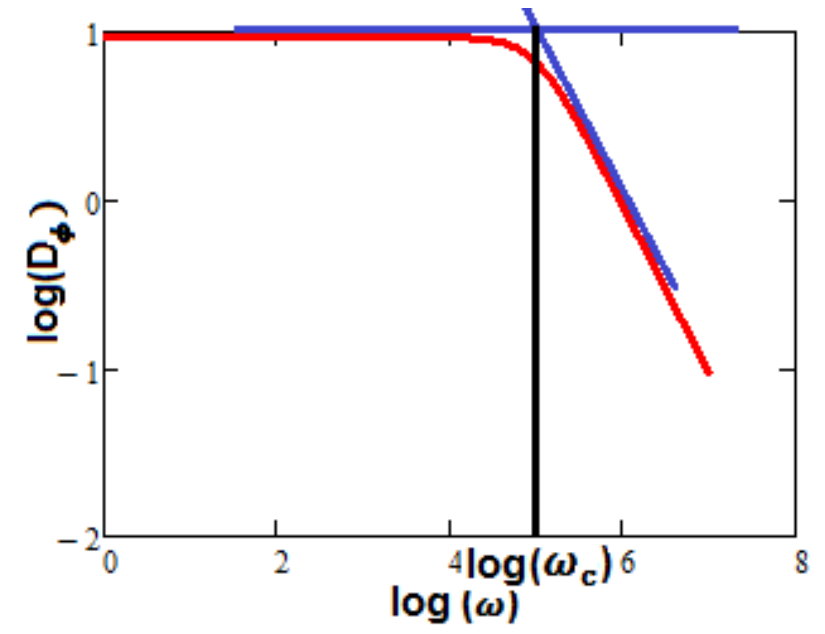

Figure 5: $\log \left(\mathrm{D}_{\phi}\right)$ according to $\log (\omega)$, $\mathrm{Kl}=15 \mathrm{~cm}^{2} \cdot \mathrm{s}^{-1}$

\section{NYQUIST DIAGRAM OF THE DIFFUSION COEFFICIENT AND ITS EQUIVALENT CIRCUITS}

The Nyquist diagram is generally a complex plan representation $[6,7,12,13,14]$ of the imaginary part versus the real one of impedance. On figure $\mathbf{6}$, the imaginary part of the diffusion coefficient versus its real part, for different values of irradiation energy is represented:

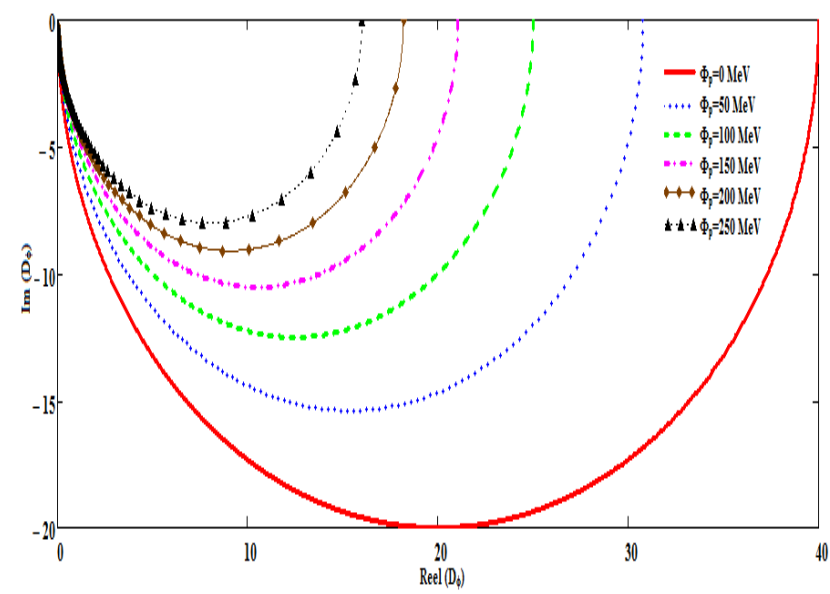

Figure 6: imaginary part versus real part of $\mathbf{D}_{\phi}$, $\mathrm{KI}=15 \mathrm{~cm}^{2} \cdot \mathrm{s}^{-1}$.

The Nyquist diagram obtained in figure 6, shows semi-circular curves [15] where $\operatorname{Im}\left(D_{\phi}\right)$ is negative. The increase of the irradiation energy has effect on the minority carriers' displacement since their diffusion coefficient decrease. For a given irradiation energy, the curve corresponds to a diameter which is compared to a parallel resistance $\mathrm{R}_{\mathrm{p}}$ as shown in figure 7 : 


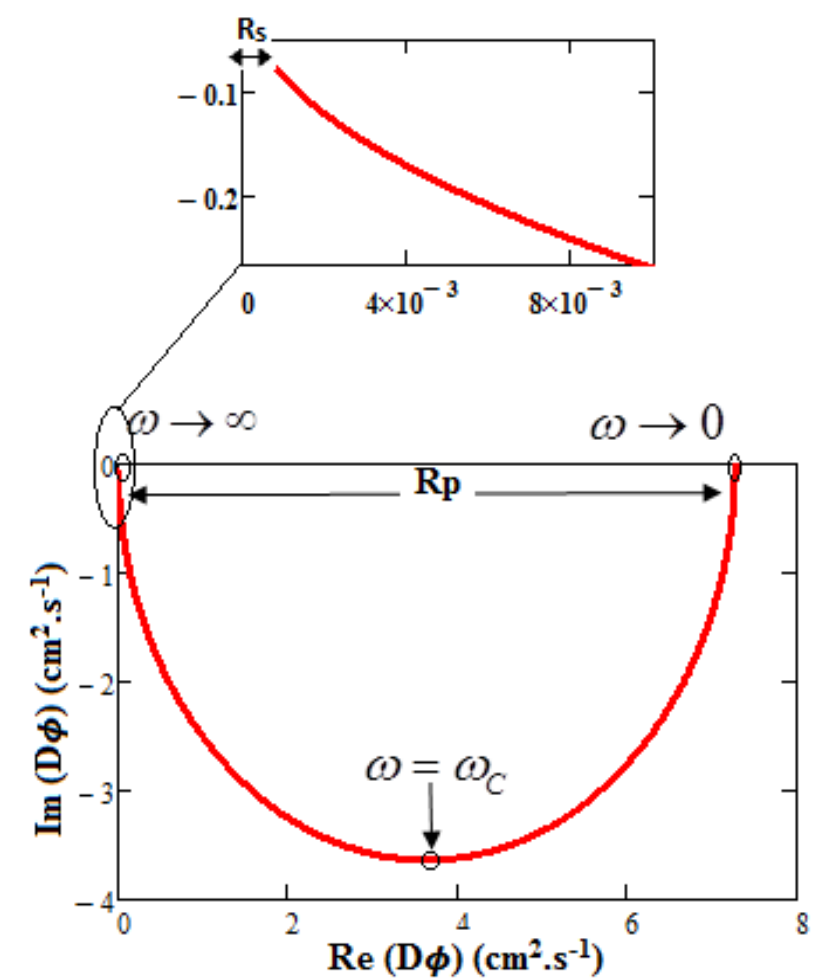

Figure 7: Diagram of Nyquist for $\mathrm{Kl}=15 \mathrm{~cm}^{2} . \mathrm{s}^{-1}$; $\phi_{\mathrm{p}}=250 \mathrm{MeV}$

This curve above, presents three characteristic points corresponding to the angular frequencies: $\omega=0, \quad \omega=\omega c$ and $\omega \rightarrow \infty$. For $\omega=0$, the imaginary part is equal to zero while the real part corresponds to a value different to zero. For $\omega=\omega c$, the imaginary part is negative but the two parts imaginary and real are different to zero. When $\omega \rightarrow \infty$, the imaginary part is equal to zero but not the real one; what enable us to determine the diameter of the half-circle and the series resistance. Starting from the Bode and Nyquist diagrams of the diffusion coefficient, an equivalent circuit describing the electric behavior of the diffusion is represented on figure $\mathbf{8}$ :

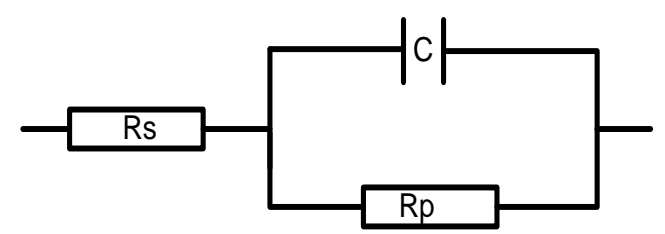

Figure 8: equivalent circuit of the diffusion coefficient Where $C$ is a capacitance, $R_{p}$ is a parallel resistance and $R_{s}$ is a serie resistance.

\section{RESULTS AND DISCUSSION}

For a value of irradiation energy, the diameter of the half-circle obtained is equal to $R_{p}[14,16]$ which is a resistance associated to the diffusion process by $\mathrm{D}_{\phi}$. From the relation $\tau=1 / \omega_{\mathrm{c}}$ for a cut-off angular frequency value, we can deduce the lifetime of the photo generated minority carriers. By the use of the time-constant $\tau=R_{p} . C$ [17], we calculate the capacitance $C$. Based on these relations, we give some results on the table below:

Table: electric and intrinsic parameters of the diffusion coefficient:

\begin{tabular}{|c|c|c|c|c|}
\hline $\begin{array}{c}\phi_{\mathrm{p}} \\
(\mathrm{MeV})\end{array}$ & $\begin{array}{r}\mathrm{R}_{\mathrm{s}} \\
(\mathrm{m} \Omega)\end{array}$ & $\begin{array}{l}\mathrm{R}_{\mathrm{P}} \\
(\Omega)\end{array}$ & $\overline{\tau(\mu \mathrm{s})}$ & $\mathrm{C}(\mu \mathrm{F})$ \\
\hline 0 & 0.99 & 10 & 63 & 628 \\
\hline 50 & 0.93 & 9.3 & 63 & 584 \\
\hline 100 & 0.87 & 8.7 & 63 & 546 \\
\hline 150 & 0.82 & 8.2 & 63 & 513 \\
\hline 200 & 0.77 & 7.7 & 63 & 483 \\
\hline 250 & 0.73 & 7.3 & 63 & 457 \\
\hline
\end{tabular}

We assume a constant lifetime of photogenerated minority carriers since the cut-off angular frequency is almost the same. When the irradiation energy increases, resistances $R_{s}$ and $R_{p}$ decrease. The capacitance $\mathrm{C}$ also decreases because there are few minority carriers stocked in the base of the solar cell. What can be explained by the fact that diffusion of the minority carriers, is slowed down. Consequently, the intrinsic properties of the photovoltaic cell are damaged, what causes poor quality and efficiency of the cell.

\section{CONCLUSION}

A theoretical study was carried out on the diffusion coefficient of a photovoltaic cell with parallel vertical junction according angular frequency and irradiation energy. We note that diffusion of the photogenerated minority carriers in the base is also governed by frequency and irradiation. Bode and Nyquist diagrams permit us to model diffusion minority carriers' phenomenon in electric one. This leads to electrical parameters determinations that depend on frequency and irradiation.

\section{REFERENCES}

[1] D. Sontag, G. Hahn, P. Geiger, P. Fath, E. Bucher, Two-dimensional resolution of minority carrier diffusion constants in different 
silicon materials, Solar Energy Materials \& Solar cells, 72 (2002) pp 533-539

[2] M. Zoungrana, I. Zerbo, A. Sere, B. Zouma, F. Zougmore 3D Study of Bifacial Silicon Solar Cell Under Intense Light Concentration and Under External Constant Magnetic Field, Global Journal of Engineering Research, vol.10, N.1\&2,(2009), pp113-124

[3] Carsten Schinke, David Hinken, Karsten Bothe, Christian Ulzhöfer, Ashley Milsted, JanSchmidt, Rolf Brendel Determination of the Collection Diffusion Length by Electroluminescence Imaging Energy Procedia, 8, (2011), pp. 147 - 152

[4] Andreas Mandelis, 'Coupled ac photocurrent and photothermal reflectance response theory of semiconducting p-n junctions," Journal of Applied Physics, Vol.66, No.11, 1989, pp. 5572-5583

[5] Lathi, Bhagwandas Pannalal: Signals, Systems and Controls. Intext Educational Publisher, New York, 1973-1974

[6] M. M. Dione, S. Mbodji, M. L. Samb, M. Dieng, M.Thiame, S. Ndoye, F. I. Barro, G. Sissoko, "Vertical junction under constant multispectral light: Determination of recombination parameters", Proceedings of the 24th European Photovoltaic Solar Energy Conference, (2009), pp.465-469

[7] Arora, J.D, S.N. Singh and P.C. Mathur, "Surface Recombination effects on the performance of $n^{+}-p$ step and diffused junction silicon solar cells", Solid State Electronics, 24(8) (1981) pp.739-747

[8] J. Philibert, Diffusion et transport de matière dans les solides, Les éditions de physique, ISBN 2-86883-004-8, (1991)

[9] N. R. Zangenberg, J. Fage-Pedersen, J. Lundsgaard Hansen, and A. N. Larsen, Boron and phosphorus diffusion in strained and relaxed Si and SiGe, J. of Appl. Phys., 94(6), 3883 (2003)

[10] H. W. Kraner, Radiation damage in silicon detectors, 2nd Pisa Meeting on Advanced Detector, Grosetto, Italy, June 3-7, 1983

[11] Amadou Diao, Ndeye Thiam, Martial Zoungrana, Gokhan Sahin, Mor Ndiaye,
Grégoire Sissoko, "Diffusion Coefficient in Silicon Solar Cell with Applied Magnetic Field and under Frequency: Electric Equivalent Circuits"; World Journal of Condensed Matter Physics, 4, (2014), pp 84 - 92

[12] Nd. Thiam, A. Diao, M. Ndiaye, A. Dieng, A. Thiam, M. Sarr, A.S. Maiga And G. Sissoko, 'Electric equivalent models of intrinsic recombination velocities of a bifacial silicon solar cell under frequency modulation and magnetic field effect,' Research Journal of Applied Sciences, Engineering and Technology, Vol.4, No.22, 2012, pp.46464655

[13] D. Chenvidhya, K. Kirtikara, C. Jivacate, "A new characterization method for solar cell dynamic impedance," Solar Energy Materials and Solar Cells, Vol.80, No.4, 2003, pp.459464

[14] R. A. Kumar, M. S. Suresh, J. Nagaraju, 'Measurement of AC parameters for Gallium Arsenide $(\mathrm{GaAs} / \mathrm{Ge})$ solar cell by impedance spectroscopy," IEEE Transactions on Electron Devices. Vol.48, No.9, 2001, pp.2177-2179

[15] Ivan Mora-Sero, Germa Garcia-Belmonte, Pablo P. Boix, Miguel A. Vazquez and Juan Bisquert, 'Impedance spectroscopy characterisation of highly efficient silicon solar cells under different light illumination intensities," Energy and Environmental Science, Vol.2, No.6, 2009, pp.678-686

[16] M. S. Suresh, 'Measurement of solar cell parameters using impedance spectroscopy," Solar Energy Materials and Solar Cells, Vol.43, Issue1, 1996, pp.21-28

[17] A DIENG, I ZERBO, M WADE, A S MAIGA AND G SISSOKO, 'Three-dimensional study of a polycrystalline silicon solar cell: the influence of the applied magnetic field on the electrical parameters", Semicond. Sci. Technol. 26 (2011) 095023 (9pp) 